\title{
A Novel Voltage Balancing Method of Modular Multilevel Converters
}

\author{
Zunfang Chu, Zixin Li, Ping Wang, Yaohua Li \\ Key Laboratory of Power Electronics and Electric Drive, Institute of Electrical Engineering, \\ Chinese Academy of Sciences, Beijing, China \\ Email: chuzf@mail.iee.ac.cn, lzx@mail.iee.ac.cn, wangping@mail.iee.ac.cn,yhli@mail.iee.ac.cn
}

Received September, 2012

\begin{abstract}
In this paper, a novel voltage balancing method of modular multilevel converters (MMCs) is proposed. This method divides the voltages of sub-module capacitors in each arm into several groups and the voltage balancing is based on these groups. The proposed method can save sorting time greatly compared with the conventional method. Simulation results on a MMC based three-phase inverter show validity of the proposed method.
\end{abstract}

Keywords: Modular Multilevel Converter; Voltage Balancing

\section{Introduction}

Modular multilevel converter (MMC) is getting more and more attention because of its characteristic of highly suitable for medium to high voltage applications. The principle of the MMC and the basic method of the voltage balancing were introduced in [1]. There are many published academic papers on MMC [2-7] recent years. There exist generally two types of PWM methods for MMC with half bridge as the sub-modules, i.e. the carrier-phase-shifted PWM (CPSPWM) method in [2,3,6] and the sub-modules unified pulse width modulated (SUPWM) method in $[4,5,7]$. The SUPWM method can balance the sub-module capacitor voltages by sorting and selecting the different sub-modules without close-loop voltage balancing controllers. With the rising of the network voltage level, the number of sub-modules in each arm is increased. So the sorting time is increased and influences the control program [6] developed a reduced switching-frequency (RSF) voltage balancing algorithm. This method significantly reduces the average device switching frequency and total switching losses of the converter. But if this method is used in SUPWM method, the voltages of the sub-module capacitors fluctuate largely. The reason is that some capacitors of sub-modules are charged (or discharged) in a half fundamental period. [8] reported a voltage-balancing technique using in multimodal converter-based high-voltage dc power transmission which employed selective harmonic elimination pulse-width-modulation (SHE-PWM). But SHW-PWM method is difficultly used in condition that the level of output voltage is more than ten.
To improve the performance of SUPWM method, this paper presents a novel method to reduce sorting time in order to balance voltage of sub-module capacitor.

\section{The Conventional Voltage Balancing Me- thod for SUPWM Method for MMC}

Figure 1 shows three phase of MMC as an inverter. It contains three phase and each phase has two arms (upper arm and lower arm). Each arm has $N$ sub-modules and one inductor $L_{0}$. A single sub-module is shown in Figure $\mathbf{1}$; it consists of one capacitor and two IGBTs. The output of sub-module $u_{S M}$ has two levels, i.e. 0 (switched off) and $U_{S M}$ (switched on). So each arm will have $N+1$ voltage levels.

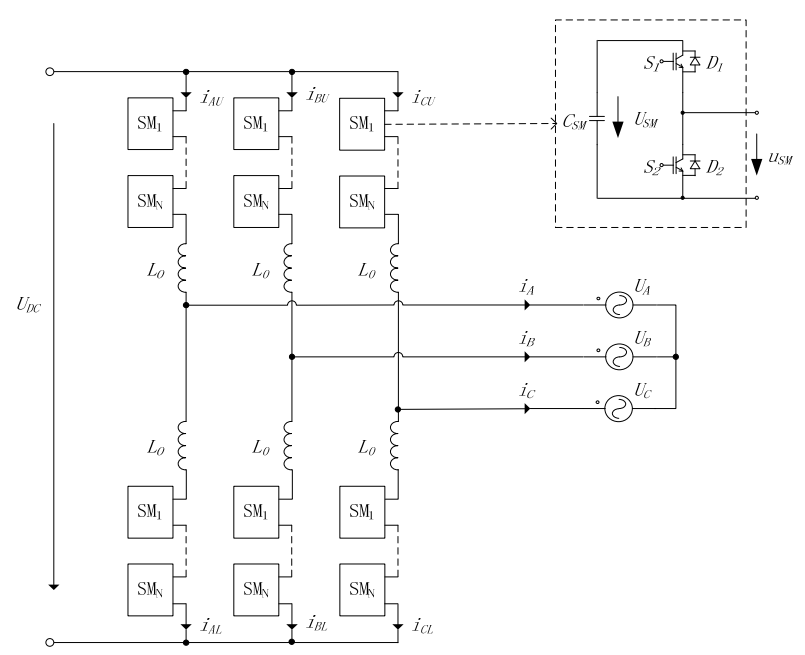

Figure 1. Three phase of MMC as an inverter. 
The basic principles of the conventional voltage balancing method are introduced in [1] as follows.

- All of voltages of sub-module capacitors in the same arm are sorted according to the order from lower to higher or from higher to lower.

- When the arm current charges the capacitors, sub-module whose capacitor voltage is lower is switched on and sub-module whose capacitor voltage is higher is switched off.

- When the arm current discharges the capacitors, sub-module whose capacitor voltage is higher is switched on and sub-module whose capacitor voltage is lower is switched off.

The conventional voltage balancing method needs to sort all capacitor voltages in the same arm each control period. If the number of sub-modules in each arm is very large, the sorting time can occupy a large proportion.

\section{The Novel Voltage Balancing Method for SUPWM Method for MMC}

The novel voltage balancing method is derived from the conventional voltage balancing method. The basic principles of the novel voltage balancing method are presented as follows.

- The sub-modules in each arm are divided into several equal groups. For example, if the number of sub-modules in each arm is 20 and the number of groups can be selected as 5 , each group can have four sub-modules. Sub-modules 1, 2, 3 and 4 can be assigned to group1, sub-modules 5, 6, 7 and 8 can be assigned to group2, sub-modules 9 , 10,11 and 12 can be assigned to group3, submodules 13, 14, 15 and 16 can be assigned to group4 and sub-modules 17, 18, 19 and 20 can be assigned to group 5.

- The voltages of sub-module comparators in each group are sorted according to the order from lower to higher or from higher to lower.

- The average voltage of each group is computed and the average voltages in each arm are sorted according to the order from lower to higher or from higher to lower.

- According to the arm current and the number of sub-modules which are needed to switch on, the groups are labeled by 1 (all of sub-modules in this group are switched on), 2 (some of sub-modules in this group are switched on) and 3 (all of sub-modules in this group are switched off). When the arm current is charging capacitors, the label of group whose average voltage is lower is 1 or 2 and the label of group whose average voltage is higher is 2 or 3 . When the arm current is discharging capacitors, the label of group whose average voltage is higher is 1 or 2 and the label of group whose average voltage is lower is 2 or 3 . For example, if the sorting result of average voltages is group 4 >group 3 >group 5 >group $1>$ group 2 , the arm current is charging the capacitors and the number of sub-modules which are needed to switch on is 10, the labels of group 2 and group1 will be 1 , the label of group 5 will be 2 and the labels of group 3 and group 4 will be 3 .

The sub-modules in each group will be switched on or off based on the sorting result of the capacitor voltages in group, the label of group, the arm current and the number of sub-modules which are needed to switch on. If the label of group is 1 (or 3), all of sub-modules in this group are switched on (or switched off). When the arm current is charging capacitors and the label of group is 2, sub-module whose capacitor voltage is lower is switched on and sub-module whose capacitor voltage is higher is switched off. When the arm current is discharging capacitors and the label of group is 2, sub-module whose capacitor voltage is higher is switched on and the sub-module whose capacitor voltage is lower is switched off. For example, if the arm current is charging capacitors, the number of sub-modules which are needed to switch on is 10 , the labels of group 2 and group 1 are 1 , the label of group 5 is 2, the labels of group 3 and group 4 are 3 and the sorting result of capacitor voltages of group 5 is $\mathrm{SM}_{20}>\mathrm{SM}_{18}>\mathrm{SM}_{17}>\mathrm{SM}_{19}$, all of sub-modules in group 2 and group 1 are switched on, all of sub-modules in group 3 and group 4 are switched off, $\mathrm{SM}_{17}$ and $\mathrm{SM}_{19}$ are switched on and $\mathrm{SM}_{20}$ and $\mathrm{SM}_{18}$ are switched off.

\section{Simulation Results}

In order to verify the validity of the proposed voltage balancing method in this paper, a MMC based threephase inverter is taken as the test example. The setup of the inverter for simulation is shown in Figure 1. Computer simulation is carried out first in PSIM software and the parameters are listed in Table $\mathbf{1 .}$

The number of group is set to four, so each group has three sub-modules. Simulated waveforms of conventional voltage balancing method are depicted in Figure 2

Table 1.

\begin{tabular}{lc}
\hline AC system voltage (line-to-line) & $U_{S}=10 \mathrm{kV}$ \\
\hline Active Power & $P=12 \mathrm{MW}$ \\
Reactive Power & $Q=6 \mathrm{MVA}$ \\
DC-link voltage & $U_{D C}=20 \mathrm{kV}$ \\
Sub-module capacitor & $C_{S M}=8.5 \mathrm{mF}$ \\
Buffer inductors & $L_{0}=8 \mathrm{mH}$ \\
No. of sub-modules in each arm & $N=12$ \\
Voltage ref. of sub-module capacitor & $U_{S M_{-} \text {ref }}=1667 \mathrm{~V}$ \\
Carrier frequency & $f_{\text {carrier }}=2 \mathrm{kHz}$ \\
\hline
\end{tabular}




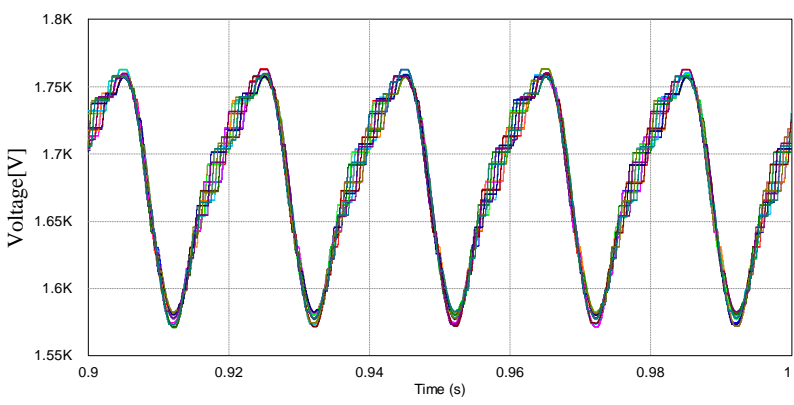

(a) Voltages of sub-module capacitors in upper arm of phase $\mathrm{A}$

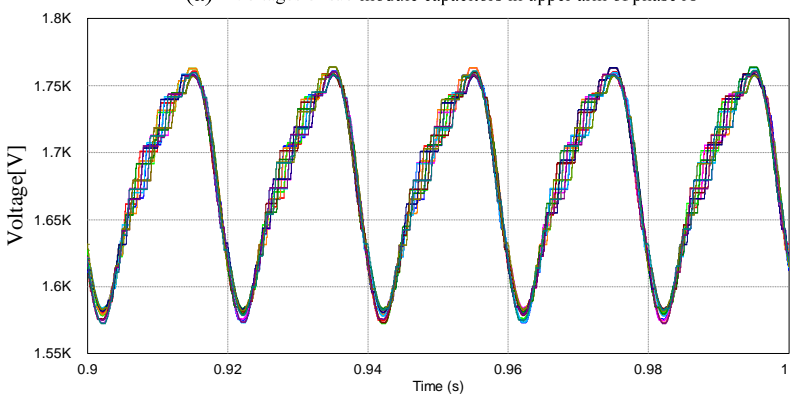

(b) Voltages of sub-module capacitors in lower arm of phase $\mathrm{A}$

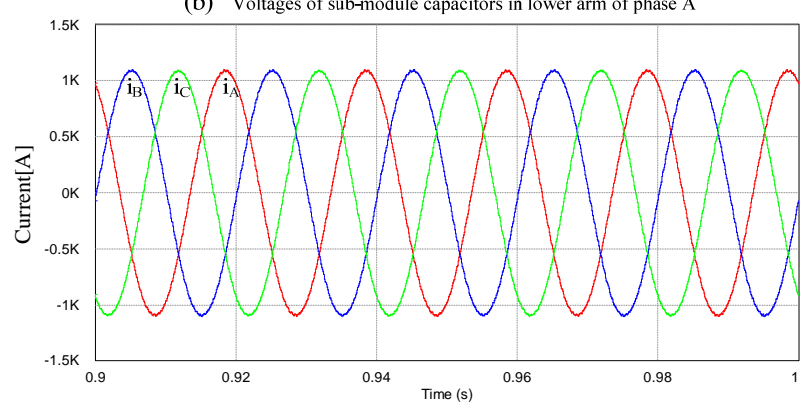

(c) Three phase currents $\left(i_{A}, i_{B}, i_{C}\right)$

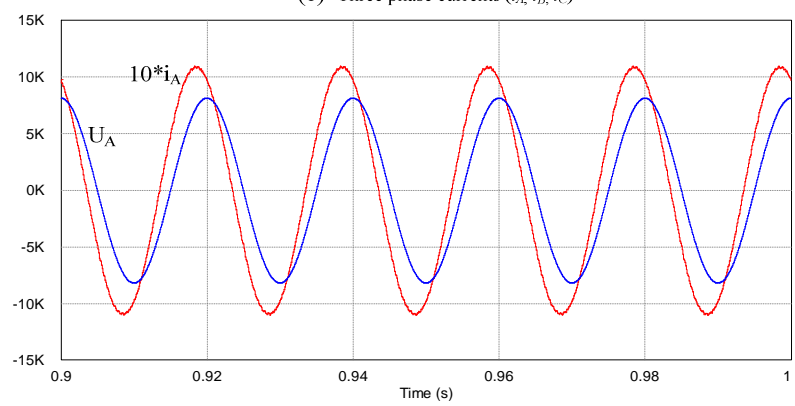

(d) Voltage of phase A and ten times current of phase A

Figure 2. Simulated waveforms of conventional voltage balancing method.

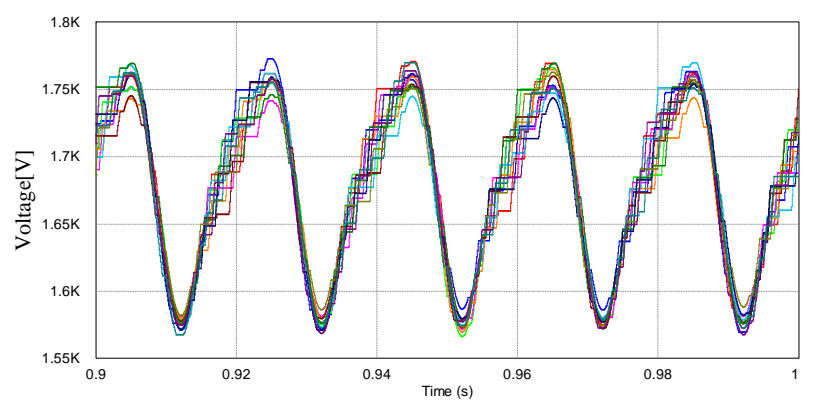

(a) Voltages of sub-module capacitors in upper arm of phase $\mathrm{A}$

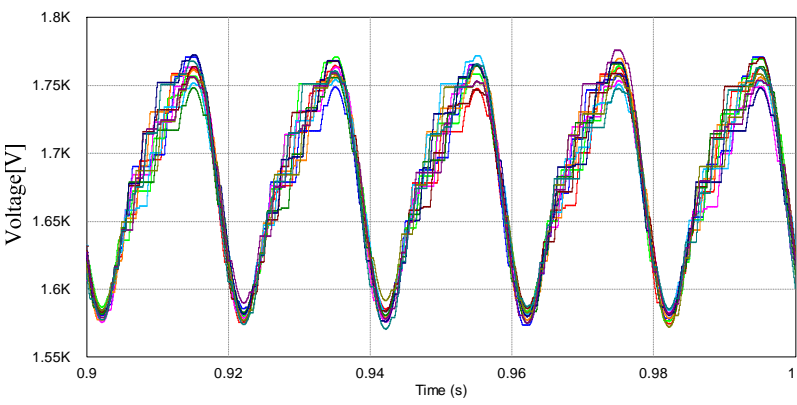

(b) Voltages of sub-module capacitors in lower arm of phase A

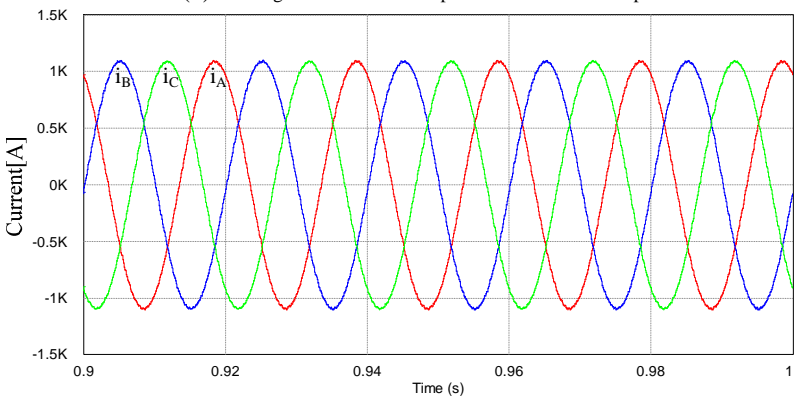

(c) Three phase currents $\left(i_{A}, i_{B}, i_{C}\right)$

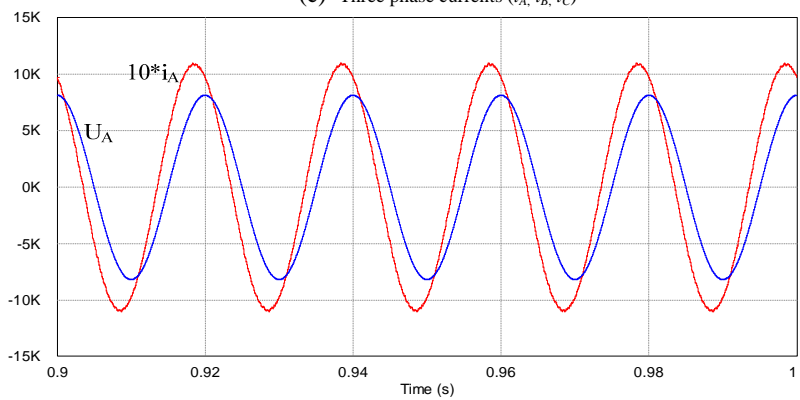

(d) Voltage of phase $\mathrm{A}$ and ten times current of phase $\mathrm{A}$

Figure 3. Simulated waveforms of proposed voltage balancing method.

and Figure 3 shows the simulation results of proposed voltage balancing method.

Compared Figure 2 with Figure 3, the voltage fluctuation range of sub-modules capacitors using proposed method is 200 larger than conventional voltage balancing method 140. From Figures 3(a) and (b), the voltages of sub-module capacitors are balanced. Although the balancing of voltage of proposed method is worse than conventional method, the sorting time of proposed method is shorter than conventional. Because the number of voltages which are needed to be sorted using conventional method is twelve and it is only four when using proposed method. So the biggest advantage of proposed method is that it greatly saves sorting time.

\section{Summary}

This paper proposed a novel voltage balancing method for MMCs. The proposed method is suitable for more sub-modules in arms. The more sub-modules each arm has, the more sorting time is saved compared with con- 
ventional method. Simulation results proved the validity of this method.

\section{REFERENCES}

[1] A. Lesnicar and R. Marquardt, “An Innovative Modular Multilevel Converter Topology Suitable for a Wide Power Range," in Power Tech Conference Proceedings, 2003 IEEE Bologna, Vol. 3, 2003, p. 6.

[2] M. Hagiwara and H. Akagi, "PWM Control and Experiment of Modular Multilevel Converters," in Power Electronics Specialists Conference, PESC 2008, IEEE, 2008, pp. 154-161.

[3] M. Hagiwara and H. Akagi, "Control and Experiment of Pulsewidth-Modulated Modular Multilevel Converters," Power Electronics, IEEE Transactions on, Vol. 24, 2009, pp. 1737-1746. doi:10.1109/TPEL.2009.2014236

[4] S. Rohner, et al., "Pulse Width Modulation Scheme for the Modular Multilevel Converter," in Power Electronics and Applications, EPE '09. 13th European Conference on,
2009, pp. 1-10.

[5] S. Rohner, et al., "Modulation, Losses, and Semiconductor Requirements of Modular Multilevel Converters," Industrial Electronics, IEEE Transactions on, Vol. 57, 2010, pp. 2633-2642.

[6] T. Qingrui, et al., "Reduced Switching-Frequency Modulation and Circulating Current Suppression for Modular Multilevel Converters," Power Delivery, IEEE Transactions on, Vol. 26, 2011, pp. 2009-2017. doi:10.1109/TPWRD.2011.2115258

[7] Z. Li, et al., "An Improved Pulse Width Modulation Method for Chopper-Cell Based Modular Multilevel Converters,” Power Electronics, IEEE Transactions on, 2012, p. 1.

[8] N. Flourentzou and V. G. Agelidis, "Multimodule HVDC System Using SHE-PWM With DC Capacitor Voltage Equalization," Power Delivery, IEEE Transactions on, Vol. 27, pp. 79-86, 2012.doi:10.1109/TPWRD.2011.2167989 